\title{
A Useful Microsoft Excel Add-in Program for Pharmacokinetic Analysis
}

\section{Baojian $\mathrm{Wu}^{*}$ and Ming $\mathrm{Hu}$}

Department of Pharmacological and Pharmaceutical Sciences, College of Pharmacy, University of Houston, 1441 Moursund Street, Houston, TX 77030, USA

\begin{abstract}
The aim of this study is to explore the possibility and versatility of Microsoft Excel in handling pharmacokinetic analysis (including model composing, simulation, and parameter estimation). We have developed the first version of an in-house pharmacokinetic tool XISimEst, coded in Visual Basic for Application (VBA), which can be used for pharmacokinetic modeling and simulation with user-tailored models consisting of a system of differential equations. The special features of the program are: (1) pharmacokinetic models can be open and saved as ordinary text files, thus the model files can be created and edited using any text editor; (2) a quicker interface to perform simulation studies; (3) capable of a standard error estimation for fitted parameters; and (4) a simple interface for generating concentration-time plots.
\end{abstract}

Keywords: Pharmacokinetic Modeling; Excel; Free program; Differential equation; XlSimEst

\section{Introduction}

As an indispensible tool in drug research, pharmacokinetic modeling provides substantial insights into the underlying mechanisms with respect to drug absorption, distribution, metabolism and excretion (ADME) in humans and other animal species. The derived key parameters such as the elimination rate constant $(\mathrm{K})$, volume of distribution $(\mathrm{V})$ and clearance $(\mathrm{CL})$ are frequently used to guide preclinical and clinical dose regimens. Furthermore, simulations based on an established pharmacokinetic model can be used to identify a rate-determining step in drug clearance (a procedure called "sensitivity analysis"), and to predict therapeutic events associated with changes in this process [1].

Mathematically, simple pharmacokinetic models can be described in an algebraic form (i.e., non-linear regression equations) or be solved by linearization methods such as Laplace transforms. However, solving complex systems using Laplace transforms tends to be tedious and inefficient. In particular, complex pharmacokinetic models involving saturable processes (e.g., metabolism and carrier-mediated transport) cannot be linearized with an analytic form, which thus require a numeric solution. As mentioned by Meineke and Brockmöller [2], coupled differential equations represent a universal approach to describe pharmacokinetic models in a more direct and effective manner.

Pharmacokinetic models specified as a set of differential equations can be handled by a variety of outstanding programs such as WinNonlin, Kinetica, and SAAM. These softwares provide a builtin model library, within which the models are generally designed for characterizing in vivo plasma concentration-time profiles. If one needs to model drug disposition in ex vivo (e.g., isolated liver perfusion) or in cellulo (e.g., Caco-2) experiments, he or she might have to build his or her own models according to a specific experimentation and a compartmentalization scheme. Although composing user-desired models is possible in abovementioned programs, learning/re-learning of this functionality tends to be difficult and time-consuming for novice or occasional users. Also, as noted by Zhang et al. [3], these softwares are very expensive, which are often not affordable to academic users. Therefore, there is a need to explore the possibility of cost-effective and easy-to-use alternative for composing desired pharmacokinetic models and performing the modeling.
Microsoft (MS) Excel spreadsheet has become the standard platform for data collection, graphing and analysis, and majority of original data are kept as MS Excel files in pharmaceutical field. The advantages of Excel include (1) the user-friendly interface and ease of use; (2) it offers many built-in mathematical and graphical routines which can be called in user-defined functions; and (3) it provides tremendous customization through macros and Visual Basic add-ins, for those users with specific needs and programming experience. By far, numerous Excel-based spreadsheet templates and add-ins have been widely programmed for data analyses in pharmaceutical sciences [46], including pharmacokinetics [2,3,7-10], suggesting the unmet needs regarding pharmacokinetics analysis with current programs. Among those works, PKsolver [3] and pk-engine [2] represent two exemplary Excel add-in programs for pharmacokinetic analysis. Although PKsolver automates pharmacokinetic analysis with a number of buildin non-linear regression models, it neither accepts user-defined models nor approximates standard errors for fitted parameters. Pk-engine is designed to simulate complex pharmacokinetic models. However, pharmacokinetic modeling (parameter estimation) is not considered in this program.

The goal of our study therefore is to provide an accurate and free program that streamlines the pharmacokinetic model composing, simulation and parameter estimation in MS Excel. In this paper, we present the first version of our add-in program XlSimEst. This program features in (1) the ease of model preparation (i.e., writing of the model), even for non-professionals, (2) a quicker interface to generate concentration-time profiles, and (3) being capable of a standard error approximation for modeled parameters. In addition, a couple of data-

\footnotetext{
*Corresponding author: Baojian Wu, 1441 Moursund Street, Department of Pharmacological and Pharmaceutical Sciences, College of Pharmacy, University of Houston, Houston, TX77030, USA Tel: (832)-531-1134; E-mail: bwu3@uh.edu

Received October 19, 2011; Accepted November 10, 2011; Published November 12, 2011

Citation: Wu B, Hu M (2011) A Useful Microsoft Excel Add-in Program for Pharmacokinetic Analysis. Pharm Anal Acta S11:002. doi:10.4172/2153-2435.S11. 002

Copyright: (c) 2011 Wu B, et al. This is an open-access article distributed under the terms of the Creative Commons Attribution License, which permits unrestricted use, distribution, and reproduction in any medium, provided the original author and source are credited.
} 
based examples were used to qualify the program and the results were compared to those derived from professional software packages (i.e., WinNonlin and ADPAT II).

\section{Implementation}

\section{Pharmacokinetic modeling}

Pharmacokinetic models here are specified as a set of differential equations (Figure 1). The Runge-Kutta method (fourth order with variable step size) is implemented to numerically solve the differential equations [11]. In an earlier study [12], this numerical integration technique was used to develop physiologically based pharmacokinetic models yielding results remarkably consistent with those from more sophisticated computer software packages.

The add-in program "XISimEst" appears as four buttons (command bars) in the region [Custom Toolbars] under Add-Ins tab in MS Excel after a quick installation, as illustrated in Figure 2. A flowchart of pharmacokinetic modeling using XlSimEst is shown in Figure 3. By clicking [XISimEst] button in the command bar, users will be prompted to specify a desired application (either simulation or parameter estimation), and to input the model (Figure 4). Pharmacokinetic models can be written using this interface consisting of three folder tabs, namely, "Initialization", "State variables" and "Parameters". "Initialization" is used to enter the values for time interval and final time. "State variables" are variables that are defined with derivatives (e.g., drug/metabolite concentration or amount) (Figure 4B). "Parameters" define the model structure and are the numbers that will be varied per simulation to observe its effects on the drug disposition processes (e.g., the first-order rate constants) (Figure 4C). "State variables" are enclosed by square brackets to distinguish them from "Parameters". After completion of the model specification, it is encouraged to save the model (as .txt file) for future use by clicking "SAVE". Alternatively, one can use text editor (e.g., Notepad) to compose the desired model, and load it by clicking "OPEN" (Figure 5 ).

An Excel spreadsheet named "Results" (by default) with the required inputs and formulas (including those for Runge-Kutta numerical integration) in the appropriate cells will be yield after clicking on the "OK" button (Figure 6). The scrollbars in the spreadsheet can be used to change the parameter values for the model parameters and the initial values (defining initial condition) for the state variables. The formula links to the scrollbars can be recovered by clicking on the [parScroll] command (Figure 2), in case that they are disabled. The maximum and minimum values attached to the scroll bars can also be changed

$$
\begin{aligned}
& \text { one-compartment two-compartment } \\
& \text { three-compartment } \\
& \frac{\mathrm{dC}_{1}}{\mathrm{dt}}=-\mathrm{K}_{10} \mathrm{C}_{1} \\
& \frac{d C_{1}}{d t}=-\left(K_{10}+K_{12}\right) C_{1}+K_{21} C_{2} \\
& \frac{\mathrm{dC}_{2}}{\mathrm{dt}}=\mathrm{K}_{12} \mathrm{C}_{1}-\mathrm{K}_{21} \mathrm{C}_{2} \\
& \frac{d C_{1}}{d t}=-\left(K_{10}+K_{12}+K_{13}\right) C_{1}+K_{21} C_{2}+K_{31} C_{3} \\
& \frac{\mathrm{dC}_{2}}{\mathrm{dt}}=\mathrm{K}_{12} \mathrm{C}_{1}-\mathrm{K}_{21} \mathrm{C}_{2} \\
& \frac{\mathrm{dC}_{3}}{\mathrm{dt}}=\mathrm{K}_{13} \mathrm{C}_{1}-\mathrm{K}_{31} \mathrm{C}_{3}
\end{aligned}
$$
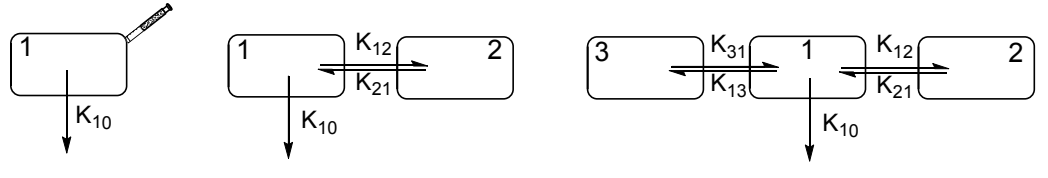

Figure 1: The one-, two-, three-compartment models describing plasma concentration-time profiles following intravenous bolus are written in the form of differential equations. $\mathrm{C}_{1}, \mathrm{C}_{2}$ and $\mathrm{C}_{3}$ are drug concentrations in the different compartments.

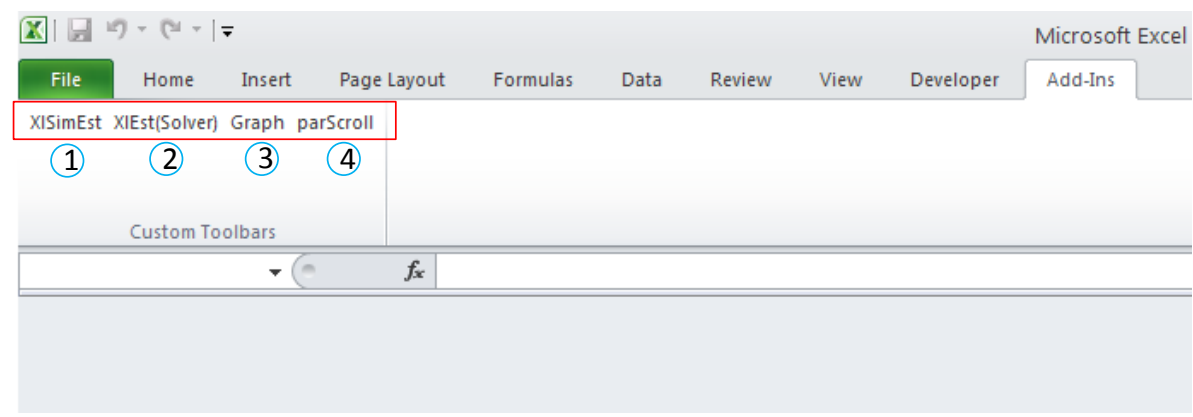

(1) Interface to generate spreadsheet for modeling and simulation

(2) Interface to perform parameter estimation using Solver

(3) Interface to generate graphs

(4) Interface to restore formula for scrollbars used to change parameter values

Figure 2: XISimEst operating buttons under Add-Ins tab in MS Excel spreadsheet . 


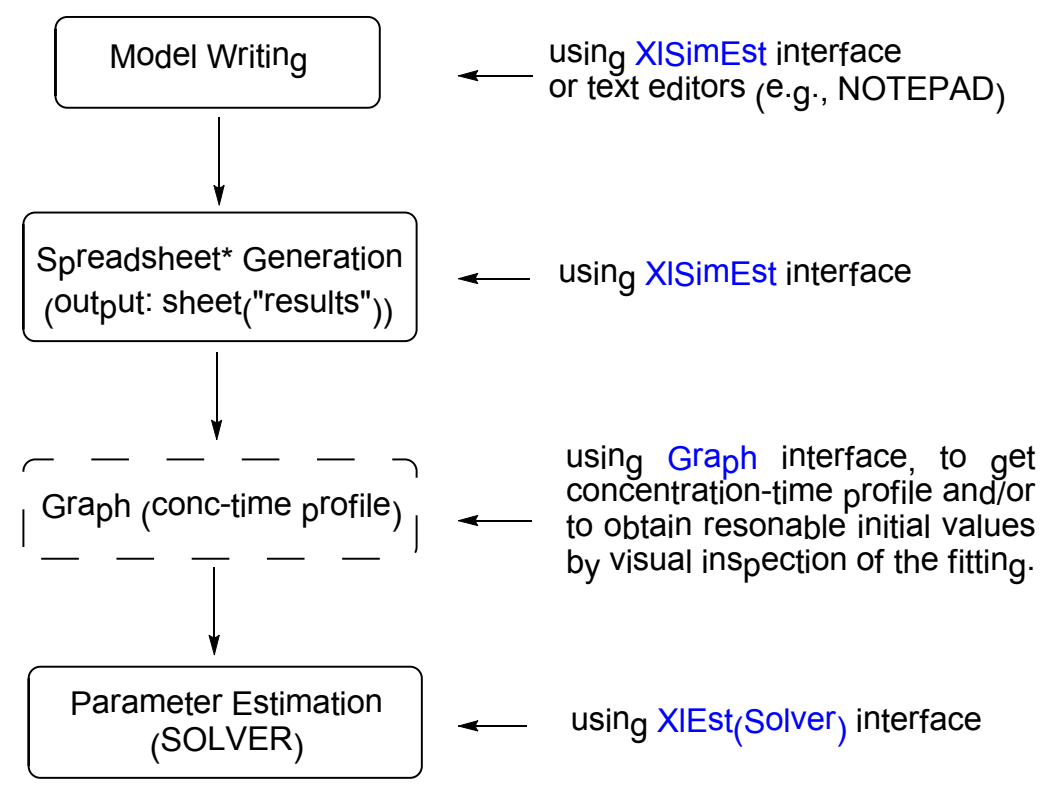

* Spreadsheet that renders a fixed-interval, fourth-order Runge-Kutta method for solving differential equations

Figure 3: The flowchart represents pharmacokinetic modeling using XISimEst.
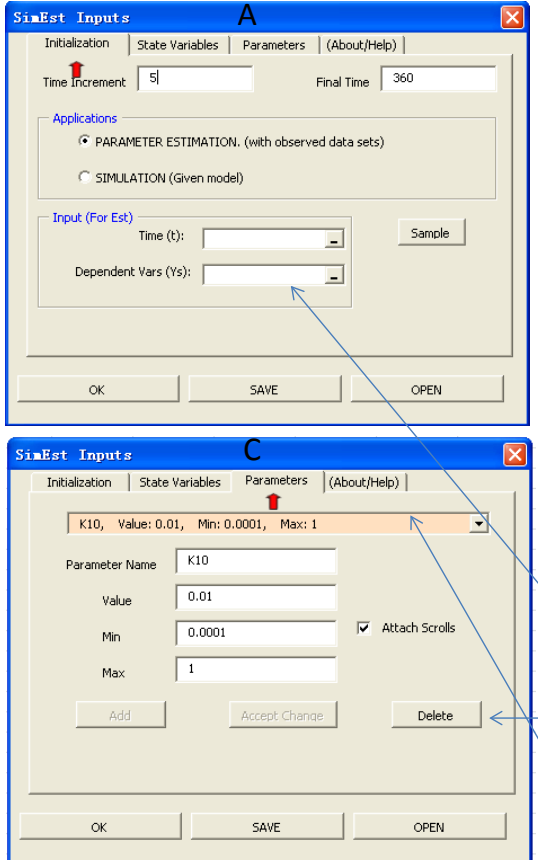

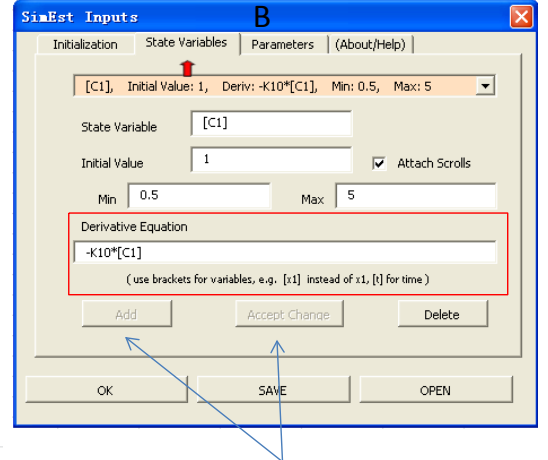

Click on the [Add] or [Accept Change] to accept the correct inputs or changes

To specify the observed data for parameter estimation, the data format is shown in "Sample". Dependent variables can be noncontinuous columns.

Click on the [Delete] to delete selected entry

Double-click on the yellow window to display a full entry

Figure 4: XISimEst interface, showing three folder tabs (i.e., "initiation", "state variables" and "parameters"). A simple model (one compartment-IV bolus, please see Fig.1) is included for demonstration. The window enclosed in box is used to write the differential equations.

manually in the spreadsheet. Fitting of the model to actual data can be visually inspected by plotting both simulated and observed values, which can be accessed by [Graph] button (Figure 7A) with "included data points" option checked. By examining how well the model approximates the data with changes in parameter values, the program provides an efficient way to obtain more reasonable initial parameter values for next parameter estimation.

Parameter optimization is performed using Excel SOLVER, which is based on the robust and reliable generalized reduced gradient 


'Time Settings
dt: 5
tfinal: 360
'State Variables
[C1], Initial Value: 1 , Deriv: $-K 10^{*}[\mathrm{C} 1]$, Min: 0.5, Max: 5
'Parameters
K10, Value: 0.01, Min: 0.0001, Max: 1

Figure 5: Code for input model of one compartment-IV bolus in NOTEPAD, a simple set of rules applies to coding of model can be found in User Manual (supplementary materials).

\begin{tabular}{|c|c|c|c|c|c|c|c|c|c|c|c|c|c|}
\hline 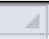 & A & B & $\mathrm{C}$ & D & E & $\mathrm{F}$ & G & $\mathrm{H}$ & I & $\mathrm{J}$ & $\mathrm{K}$ & L & M \\
\hline 1 & & \multirow{3}{*}{\multicolumn{3}{|c|}{$\begin{array}{l}\text { Select parameter cells (name } \\
\text { and values) to optimize }\end{array}$}} & & & & & & & & & \\
\hline 2 & & & & & & $\mathrm{dt}$ & 5 & & & & & & \\
\hline 3 & & & & & & Final $t:$ & 360 & & & & & & \\
\hline 4 & & & & & Parameters: & K10: & 0.01 . & 1 & Min: & 0.0001 & Max: & 1 & \\
\hline 5 & & & & & Initial Values & [A1]: & & 11 & Min: & 0.5 & Max: & 5 & \\
\hline 6 & & & & & Derivatives: & $\mathrm{d}[\mathrm{Al}] / \mathrm{dt}=$ & $-\mathrm{K} 10 *[\mathrm{H} 1]$ & & & & & & \\
\hline 7 & & & & & & & & & & & & & \\
\hline 8 & \multicolumn{4}{|c|}{ Data and Error Analysis $(\mathrm{W}=1)$} & & \multicolumn{2}{|c|}{ Simulation Results } & & & & & & \\
\hline 9 & Time & [A1] & {$[\mathrm{A} 1]^{*}$} & $\mathrm{~d}[\mathrm{~A} 1]^{\wedge} 2$ & $\mathrm{dt}$ & Time & [A1] & d1,1 & $\mathrm{d} 2,1$ & d3,1 & $\mathrm{d} 4,1$ & & \\
\hline 10 & 5 & 1.625 & 0.951229 & 0.453967 & & 0 & 1 & -0.05 & -0.04875 & -0.04878 & -0.04756 & & \\
\hline 11 & 10 & 1. 384 & 0.904837 & 0.229597 & 5 & 5 & 0.951229 & -0.04756 & -0.04637 & -0.0464 & -0.04524 & & \\
\hline 12 & 15 & 1.28 & 0.860708 & 0.175806 & 5 & 10 & 0.904837 & -0.04524 & $=-0.04411$ & -0.04414 & -0.04303 & & \\
\hline 13 & 20 & 1. 105 & 0.818731 & 0.08195 & 5 & 15 & 0.860708 & -0.04304 & $=-0.04196$ & -0.04199 & -0.04094 & & \\
\hline 14 & 30 & 0.973 & 0.740818 & 0.053908 & 5 & 20 & 0.818731 & -0.04094 & -0.03991 & -0.03994 & -0.03894 & & \\
\hline 15 & 45 & 0.806 & 0.637628 & 0.028349 & 5 & 25 & 0.778801 & -0.03894 & $=-0.03797$ & -0.03799 & -0.03704 & & \\
\hline 16 & 60 & 0.74 & 0.548812 & 0.036553 & 5 & 30 & 0.740818 & -0.03704 & $=-0.03611$ & -0.03614 & -0.03523 & & \\
\hline 17 & 90 & 0.582 & 0.40657 & 0.030776 & 5 & 35 & 0.704688 & -0.03523 & -0.03435 & -0.03438 & -0.03352 & & \\
\hline 18 & 120 & 0.53 & 0.301194 & 0.052352 & 5 & 40 & 0.67032 & -0.03352 & -0.03268 & -0.0327 & -0.03188 & & \\
\hline
\end{tabular}

Figure 6: The resulting spreadsheet for the parameter estimation.

method (GRG) [13,14]. The optimization algorithm requires the preset initial parameter values to start an iterative process. The iterative calculation stops and provides the solutions, when the target (i.e., weighted sum squares of residuals) converges at a predefined value. A detailed illustration of this SOLVER implementation in Excel is reported previously [6,15]. For the ease of use, optimization for selected parameters (either parameters or initial conditions for state variables) is performed via an interactive Excel UserForm by clicking on [XlEst(Solver)] command bar, where both parameter inputs and modeling settings are specified (Figure 7B). Users are allowed to use different weighting schemes $\left(1,1 / \mathrm{y}\right.$ and $\left.1 / \mathrm{y}^{2}\right)$ and adjust the SOLVER features such as minimization method, precision, convergence and iterations (Figure $7 \mathrm{~B}$ ). The parameter estimation is started instantaneously by clicking the "Run" button. The massive calculations then proceed in the current sheet "Result", where the estimated parameters, their respective standard errors, and model diagnostics will be presented (Figure 8). In order to facilitate a quick experience and learning of the program, sample data are included, which can be invoked simply by clicking the "Sample" button (Figure $4 \mathrm{~A}$ ). Additionally, example model files used in this paper can be found in supplemental files (User Manual).

One dataset (i.e., the abovementioned sample data) from a published book [16] was used to evaluate XISimEst. This same set of concentration-time data were fitted with one-, two-, and threecompartment IV bolus models, respectively (Figure 1). Table 1 summarizes the modeling results using XISimEst and two commercial softwares (WinNonlin and ADAPT II). All parameters and their standard errors obtained with XlSimEst are similar to those calculated with WinNonlin and ADAPT II. Moreover, the diagnostic parameters are nearly identical, which suggests that the results of XISimEst are acceptable.

\section{Model discrimination}

For model selection, XISimEst provides statistics for goodness-offit assessment such as determination of coefficient $\left(R^{2}\right)$, weighted sum squares of residuals (SS), standard error of weighted residuals (SE), Akaike's information criterion (AIC) and Schwarz criterion (SC). Among those statistics, AIC and SC include a penalty term for the number of modeled parameters [17], and are computed the same way as described earlier $[3,6]$.

\section{Standard error of the estimated parameters}

Although being capable of estimating the parameters, SOLVER does not approximate the standard errors of the estimated parameters. A special macro therefore is encoded in XISimEst to provide the standard errors with respective to the parameters resulted from SOLVER. 


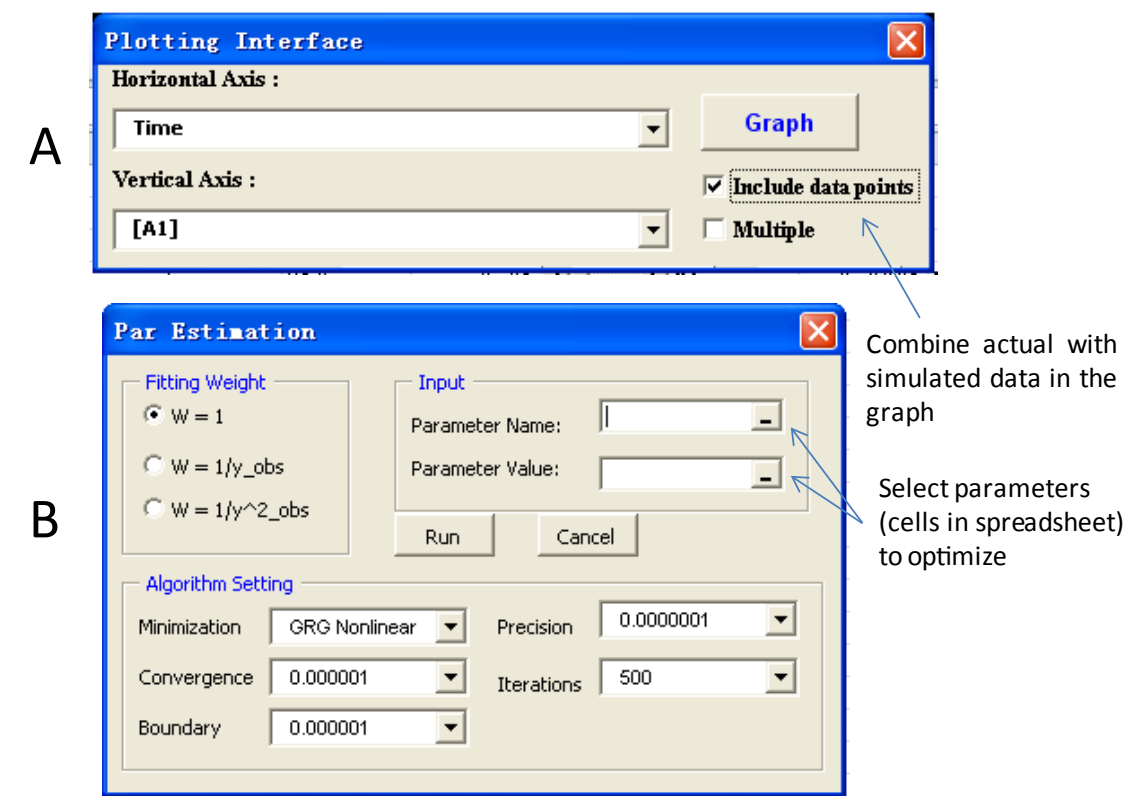

Figure 7: Panel A: a plotting interface appears after clicking on [Graph] command button; Panel B: "XISim(Solver)" interface for parameter estimation, showing SOLVER configuration and weighting schemes.

A

\begin{tabular}{|c|c|c|c|c|c|c|c|c|c|c|c|c|c|c|}
\hline 4 & A & B & C & D & E & $\mathrm{F}$ & G & $\mathrm{H}$ & & & IS & $L$ & III & \\
\hline 1 & 0.259775496 & & & & & & & & Diagnositics & $\mathbf{R}^{n} 2$ & ISS & SE & $\triangle I C$ & $\mathrm{SC}$ \\
\hline 2 & & & & & & $d t$ & 5 & & Value & 0.913584 & 0.259775 & 0.147132 & -14.8711 & -13.593 \\
\hline 3 & Paraneter & Value & Std. Dev & & & Final $t$ : & 360 & & & & & & & \\
\hline 4 & K10: & 0.007893 & 0.001148 & & Parameters: & K10: & 0.007893 & 1 & Min: & $0.0001 \mathrm{l}$ & Max: & 1 & & \\
\hline 5 & [A1]: & 1.402874 & 0.082748 & & Initial Values & [A1]: & 1. 402874 & $1]$ & Min: & $0.5 \mathrm{I}$ & Max: & 5 & & \\
\hline 6 & & & & & Derivatives: & $\mathrm{d}[\mathrm{A} 1] / \mathrm{dt}=-$ & $-\mathrm{K} 10 *[\mathrm{~A} 1]$ & & & & & & & \\
\hline 7 & & & & & & & & & & & & & & \\
\hline 8 & Data anc & d Error An & nalysis (W: & $=1)$ & & Simulatior & n Results & & & & & & & \\
\hline 9 & Time & [A1] & {$[\mathrm{A} 1]^{*}$} & $\mathrm{~d}[\mathrm{~A} 1]^{\wedge} 2$ & $\mathrm{dt}$ & Time & [A1] & $\mathrm{d} 1,1$ & $\mathrm{~d} 2,1$ & d3,1 & $\mathrm{d} 4,1$ & & & \\
\hline 10 & 5 & 1.625 & 1.348587 & 0.076404 & & 0 & 1.402874 & -0.05537 & -0.054273183 & -0.05429 & -0.05322 & & & \\
\hline 11 & 10 & 1.384 & 1. 2964 & 0.007674 & 5 & 5 & 1. 348587 & -0.05322 & -0.052172959 & -0.05219 & -0.05116 & & & \\
\hline
\end{tabular}

B

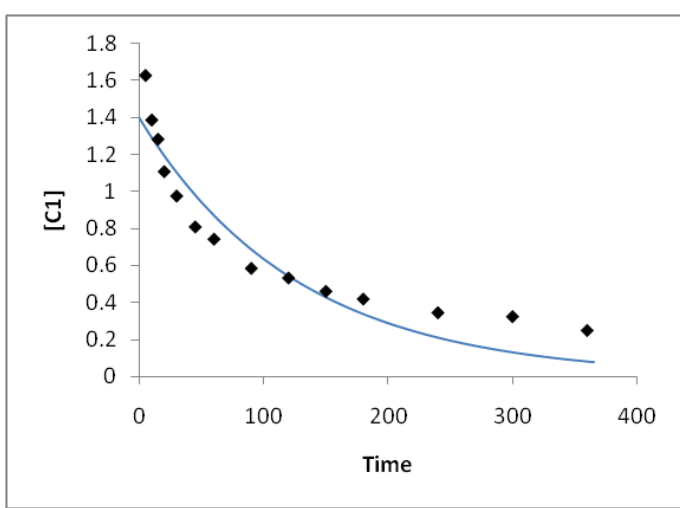

Figure 8: Panel A: Fitting results including estimated parameters and diagnostics generated from XISimEst using one-compartment-IV bolus model. Panel B: a corresponding plot of the simulation result together with raw data.

The standard error of the parameter $\mathrm{a}_{1}$ is given by [18]

$\sigma_{\mathrm{i}}=\sqrt{\mathrm{P}_{\mathrm{ii}}^{-1}} \times \mathrm{SE}$

where $P_{i i}^{-1}$ is the $i^{\text {th }}$ diagonal element of the inverse of the $P_{i j}$ matrix, $S E$ is standard error of weighted residuals.

$$
\mathrm{P}_{\mathrm{ij}}=\sum_{\mathrm{n}=1}^{\mathrm{N}} \frac{\delta \mathrm{F}_{\mathrm{n}}}{\delta \mathrm{a}_{\mathrm{i}}} \frac{\delta \mathrm{F}_{\mathrm{n}}}{\delta \mathrm{a}_{\mathrm{j}}}
$$

$\mathrm{N}$ is the number of observations. $\delta \mathrm{F}_{\mathrm{n}} / \delta \mathrm{a}_{\mathrm{i}}$ is the partial derivative of the function with respective to $a_{i}$ evaluated at $x_{n}$ ( $x$, independent variables). 
Citation: Wu B, Hu M (2011) A Useful Microsoft Excel Add-in Program for Pharmacokinetic Analysis. Pharm Anal Acta S11:002. doi:10.4172/21532435.S11-002

Page 6 of 7

\begin{tabular}{|c|c|c|c|c|c|c|c|c|c|}
\hline \multirow[b]{2}{*}{ Parameters } & \multicolumn{3}{|c|}{ One-compartment model } & \multicolumn{3}{|c|}{ Two-compartment model } & \multicolumn{3}{|c|}{ Three-compartment model } \\
\hline & XISimEst ${ }^{* \#}$ & WinNonlin & ADAPT II* & XISimEst ${ }^{\star \#}$ & WinNonlin & ADAPT $\|^{*}$ & XISimEst ${ }^{* \#}$ & WinNonlin & ADAPT II* \\
\hline $\mathrm{K}_{10}$ & $0.0079 \pm 0.0011$ & $\begin{array}{c}0.0079 \pm \\
0.0011\end{array}$ & $\begin{array}{c}0.0079 \pm \\
0.0011\end{array}$ & $0.0071 \pm 0.0004$ & $\begin{array}{c}0.0071 \pm \\
0.0004\end{array}$ & $\begin{array}{c}0.0071 \pm \\
0.0004\end{array}$ & $0.0067 \pm 0.0010$ & $\begin{array}{c}0.0067 \pm \\
0.0009\end{array}$ & $\begin{array}{c}0.0067 \pm \\
0.0009\end{array}$ \\
\hline $\mathrm{K}_{12}$ & & & & $0.0218 \pm 0.0021$ & $\begin{array}{c}0.0219 \pm \\
0.0021\end{array}$ & $\begin{array}{c}0.0219 \pm \\
0.0021\end{array}$ & $0.0194 \pm 0.0061$ & $\begin{array}{c}0.0195 \pm \\
0.0063\end{array}$ & $\begin{array}{c}0.0196 \pm \\
0.0062\end{array}$ \\
\hline $\mathrm{K}_{21}$ & & & & $0.0222 \pm 0.0028$ & $\begin{array}{c}0.0222 \pm \\
0.0028\end{array}$ & $\begin{array}{c}0.0222 \pm \\
0.0028\end{array}$ & $0.0632 \pm 0.0436$ & $\begin{array}{c}0.0632 \pm \\
0.0457\end{array}$ & $\begin{array}{c}0.0635 \pm \\
0.0457\end{array}$ \\
\hline $\mathrm{K}_{13}$ & & & & & & & $0.0121 \pm 0.0073$ & $\begin{array}{c}0.0121 \pm \\
0.0073\end{array}$ & $\begin{array}{c}0.0123 \pm \\
0.0073\end{array}$ \\
\hline $\mathrm{K}_{31}$ & & & & & & & $0.0117 \pm 0.0070$ & $\begin{array}{c}0.0117 \pm \\
0.0070\end{array}$ & $\begin{array}{c}0.0118 \pm \\
0.0070\end{array}$ \\
\hline $\mathrm{C}_{0}$ & $1.4028 \pm 0.0827$ & $\begin{array}{l}1.4027 \pm \\
0.0827\end{array}$ & $\begin{array}{c}1.4027 \pm \\
0.0827\end{array}$ & $1.8408 \pm 0.0412$ & $\begin{array}{c}1.8402 \pm \\
0.0412\end{array}$ & $\begin{array}{c}1.8402 \pm \\
0.0412\end{array}$ & $1.9271 \pm 0.0754$ & $\begin{array}{c}1.9307 \pm \\
0.0778\end{array}$ & $\begin{array}{c}1.9311 \pm \\
0.0769\end{array}$ \\
\hline \multicolumn{10}{|l|}{ Diagnostics } \\
\hline $\mathrm{R}^{2}$ & 0.9135 & 0.9135 & 0.9135 & 0.9974 & 0.9974 & 0.9974 & 0.9985 & 0.9985 & 0.9985 \\
\hline SS & 0.2598 & 0.2598 & 0.2598 & 0.0065 & 0.0065 & 0.0065 & 0.0037 & 0.0037 & 0.0037 \\
\hline SE & 0.1471 & 0.1471 & 0.1471 & 0.0255 & 0.0255 & 0.0255 & 0.0214 & 0.0214 & 0.0214 \\
\hline $\mathrm{AIC}$ & -14.87 & -14.87 & -14.87 & -62.53 & -62.53 & -62.53 & -66.47 & -66.47 & -66.47 \\
\hline SC & -13.59 & -13.59 & -13.59 & -59.98 & -59.98 & -59.98 & -62.64 & -62.64 & -62.64 \\
\hline
\end{tabular}

*Initial values were arbitrary assigned: $\mathrm{K}_{10}=\mathrm{K}_{12}=\mathrm{K}_{21}=\mathrm{K}_{13}=\mathrm{K}_{31}=0.01 ; \mathrm{C}_{0}=1$. Weighting scheme (W=1) was used in all cases. ${ }^{\#}$ step size of 5 (mins) was used for numerical integration.

Table 1: Detailed comparison of XISimEst results with those of WinNonlin 3.3 and ADAPT II.

\section{Availability}

The XISimEst package is available in Supplementary Materials. The package contains the XISimEst program file and a complete User Manual. Interested users can download and install it for free. The package was developed and tested in Excel 2010 under Microsoft Windows XP service pack 2. The program is also successfully run in Excel 2007. Other Excel (e.g., Excel 2003) or Windows versions are not tested, thus their compatibility with XlSimEst is not guaranteed. We are looking forward to receiving any suggestions from our program users.

\section{Limitations}

Since parameter optimization in XlSimEst essentially is an MS Excel SOLVER implementation, all limitations of SOLVER is also applied to this add-in program. For example, (1) the greater the number of parameters in the model, the longer the program will take; (2) the number of observations in total cannot exceed the limit of 200. It is also noted that the program is sensitive to given initial parameter value. If the initial parameters are inappropriate, SOLVER either proceeds in the wrong direction and a solution cannot found, or converges at a local minimum and provides a biased solution.

\section{Conclusion}

This study presents XlSimEst, a free MS Excel add-in program, which aims to streamline pharmacokinetic model composing, simulation and parameter estimation. As scientists are more educated in application of MS Excel, the add-in program admittedly is much user-friendly. Although the program is satisfactory regarding the estimated parameters and their respective standard errors for a limited number of example runs, further testing is required for additional models and datasets.

\section{Acknowledgement}

It is gratefully acknowledged that configuration of spreadsheet for result output and Excel UserForms in this work had been adapted from the project of RK4 by Dr. Co, which is an outstanding exemplary of implementing Runge-Kutta method in MS Excel. The author also thanks reviewers for valuable suggestions and comments.

\section{References}

1. Kusuhara H, Sugiyama $Y(2009)$ In vitro-in vivo extrapolation of transportermediated clearance in the liver and kidney. Drug Metab Pharmacokinet 24 37-52.

2. Meineke I, Brockmöller J (2007) Simulation of complex pharmacokinetic models in Microsoft Excel. Comput Methods Programs Biomed 88: 239-245.

3. Zhang Y, Huo M, Zhou J, Xie S (2010) PKSolver: An add-in program for pharmacokinetic and pharmacodynamic data analysis in Microsoft Excel. Comput Methods Programs Biomed 99: 306-314.

4. Langenbucher F (2005) Handling of computational in vitro/in vivo correlation problems by Microsoft Excel: IV. Generalized matrix analysis of linear compartment systems. Eur J Pharm Biopharm 59: 229-235.

5. Langenbucher $F(2007)$ Handling of computational in vitro/in vivo correlation problems by Microsoft Excel: V. Predictive absorbability models. Eur J Pharm Biopharm 67: 293-299.

6. Zhang Y, Huo M, Zhou J, Zou A, Li W, et al. (2010) DDSolver: an add-in program for modeling and comparison of drug dissolution profiles. AAPS $\mathrm{J} 12$ 263-271.

7. Delboy H (1994) A non-linear fitting program in pharmacokinetics with Microsoft Excel spreadsheet. Int J Biomed Comput 37: 1-14.

8. Haddad S, Pelekis M, Krishnan K (1996) A methodology for solving physiologically based pharmacokinetic models without the use of simulation 
Citation: Wu B, Hu M (2011) A Useful Microsoft Excel Add-in Program for Pharmacokinetic Analysis. Pharm Anal Acta S11:002. doi:10.4172/21532435.S11-002

softwares. Toxicol Lett 85: 113-126.

9. Sato H, Sato S, Wang YM, Horikoshi I (1996) Add-in macros for rapid and versatile calculation of non-compartmental pharmacokinetic parameters on Microsoft Excel spreadsheets. Comput Methods Programs Biomed 50: 43-52.

10. Dansirikul C, Choi M, Duffull SB (2005) Estimation of pharmacokinetic parameters from non-compartmental variables using Microsoft Excel. Comput Biol Med 35: 389-403.

11. Davies RG (1971) Computer Programming In Quantitative Biology. London/ New York: Academic Press 388.

12. Marino DJ (2005) Physiologically based pharmacokinetic modeling using microsoft excel and visual basic for applications. Toxicol Mech Methods 15: 137-154.

13. Frontline Systems, developers of the Excel Solver.
14. Lasdon LS, Waren AD, Jain A, Ratner M (1978) Design and Testing of a Generalized Reduced Gradient Code for Nonlinear Programming, ACM Trans. Mathematical Software 4: 34-50.

15. Brown AM (2001) A step-by-step guide to non-linear regression analysis of experimental data using a Microsoft Excel spreadsheet. Comput Methods Programs Biomed 65: 191-200.

16. Gabrielsson J, Weiner D (2006) Pharmacokinetic and Pharmacodynamic Data Analysis: Concepts and Applications, 4rd ed. Sweden: Swedish Pharmaceutical Press 571-576.

17. Ludden TM, Beal SL, Sheiner LB (1994) Comparison of the Akaike Information Criterion, the Schwarz criterion and the $F$ test as guides to model selection. $J$ Pharmacokinet Biopharm 22: 431-445.

18. Billo EJ (2001) Excel for Chemists: A Comprehensive Guide, $2^{\text {nd }}$ ed New York: Wiley. 